\title{
Defining the expression and function of COQ8B in TAA
}

\author{
Christian C. Cuevas ${ }^{1}$, Stephanie M. Ware ${ }^{2}$, Benjamin J. Landis ${ }^{2}$ \\ ${ }^{1}$ Indiana University School of Medicine, ${ }^{2}$ Indiana University School of Medicine, \\ Departments of Pediatrics and Medical and Molecular Genetics
}

\section{Background and Hypothesis:}

Thoracic aortic aneurysm (TAA) is characterized by progressive enlargement of the aorta and predisposes to life-threatening aortic dissection. Even among patients with monogenic causes of TAA, variable expressivity and incomplete penetrance hinders clinical decisions. Recent studies identify the gene COQ8B, a key mediator of mitochondrial ubiquinone biosynthesis, as a candidate genetic modifier in TAA. First, we sought to describe the distribution and morphological appearance of mitochondria in aortic smooth muscle cells (SMCs). We further hypothesized that decreased expression of COQ8B alters the contractile homeostasis of SMCs and predisposes to SMC apoptosis.

\section{Experimental Design or Project Methods:}

We cultured SMCs directly from aortic tissues acquired at the time of aortic replacement surgery or heart transplantation. Mitochondria were labeled in live and fixed SMCs using the MitoTracker dye. We knocked down expression of COQ8B in SMCs using siRNA (siCOQ8B). The effects of siCOQ8B were compared with SMCs treated with a non-targeting negative control siRNA (siNeg). RNA expression was measured using quantitative polymerase chain reaction (qPCR) at 48 to 72 hours after siRNA transfection. Apoptosis was measured using a TUNEL-based fluorescent assay.

\section{Results:}

Mitochondria in aortic SMCs showed a predominantly perinuclear distribution along with the appearance of a network of thin, filamentous extensions outward from the nucleus. Compared with siNeg controls, siCOQ8B decreased COQ8B RNA levels by approximately $90 \%$ on average across 5 knockdown experiments (80 to 95\% knockdown). siCOQ8B led to increased RNA levels of CNN1 across all experiments, ranging from 1.4 to 7.4 -fold increases. Meanwhile, siCOQ8b led to lower levels of COL1A1 in 4 of 5 experiments, ranging from 1.1 to 1.9-fold decreases. siCOQ8B led to decrease in the percentage of apoptotic cells in comparison with siNeg. In the presence of $1.6 \mathrm{mM}$ of $\mathrm{H}_{2} \mathrm{O}_{2}$, there was a $15 \%$ increase in apoptosis in cells transfected with siCOQ8B, and a 4.5\% increase in cells not treated with $\mathrm{H}_{2} \mathrm{O}_{2}$.

\section{Conclusion and Potential Impact:}

Decreased expression of COQ8B via siRNA knockdown alters the expression of genes important for SMC homeostasis, causing the SMC to be more susceptible to oxidative stress and inducing apoptosis. Future studies will further explore the 
role of COQ8B on SMC contractility, matrix production, and apoptosis and elucidate the underlying mechanism. Ultimately, this insight may lead to novel clinical approaches and therapies for patients with TAA. 